\title{
Assoziation von Hämorrhoiden und chronisch venöser Insuffizienz
}

Ekici U et al. Association Between Hemorrhoids and Lower Extremity Chronic Venous Insufficiency. Cureus 2019; 11(4): e4502. doi:10.7759/cureus.4502

Hämorrhoiden sind häufig, bleiben aber oft asymptomatisch. Studien aus Krankenhäusern zeigten, dass $86 \%$ der Untersuchten betroffen waren. Das Geschlecht und sozioökonomische Faktoren beeinflussen die Inzidenz nicht. Die Schwächung des Bandapparates ab der dritten Lebensdekade kann eine Protrusion innerer Hämorrhoiden verursachen. Damit verbunden ist eine Druckerhöhung auf die analen Gefäßpolster bei der Stuhlpassage, die eine vaskuläre Kongestion und Blutungen nach sich ziehen kann. Inflammatorische Prozesse induzieren die manifeste Erkrankung. Als Risikofaktoren gelten Konditionen mit erhöhtem intraabdominellem Druck: u.a. Obstipation, Diarrhoe, Schwangerschaft, Übergewicht und längeres Stehen oder Sitzen. Die Verschiebung der Altersspitze in den mittleren Bereich legte die Vermutung nahe, dass auch Ernährungsgewohnheiten einen bedeutsamen Einfluss haben. Für Varizen sind teilweise überlappende Risikofaktoren bekannt (u. a. Schwangerschaft, Alter, Übergewicht, langes Sitzen). In der prospektiven Studie überprüften Ekici et al., wie oft Patienten mit Hämorrhoiden auch eine chronisch venöse Insuffizienz (CVI) der Beine aufweisen und verglichen die Inzidenz mit einer Kontrollgruppe. Die Befunde wurden mit der CEAP-Klassifikation beurteilt.

In der Studiengruppe waren 100 Patienten, die zur Operation von inneren und äußeren Hämorrhoiden (Grad 3-4) in das Krankenhaus kamen. 100 Kontrollpersonen ohne Hämorrhoiden unterschieden sich nicht wesentlich hinsichtlich der klinischen und soziodemografischen Basisdaten. Das Alter betrug 36,89 bzw. 36,83 Jahre und der Body Mass Index 26, 17 bzw. 26,22 kg/m² (jeweils median). Keine Unterschiede bestanden auch für Schwangerschaften/Entbindungen, chronische Krankheiten, langes
Stehen/Sitzen. Verglichen mit der Kontrollgruppe wiesen Patienten mit Hämorrhoiden deutlich häufiger eine Varicosis auf (35\% vs. 19\%). Die Inzidenz von C1- und C2-Varizen war in der Studiengruppe signifikant gesteigert ( $p=0,039)$. Gleichzeitig kam eine Obstipation bei Patienten mit Hämorrhoiden öfter vor. 78 \% gegenüber $29 \%$ der Kontrollen waren betroffen ( $p<0,001)$. Die höhere Inzidenz der Varicosis bei Patienten mit Hämorrhoiden spricht für ähnliche ätiologische Faktoren. Die chronische Obstipation stufen die Autoren als gemeinsamen Trigger für die CVI und Hämorrhoiden ein. Die Literaturrecherche habe keine Hinweise auf einen direkten Zusammenhang zwischen Obstipation und CVI ergeben, aber die Koinzidenz von CVI und Hämorrhoiden spricht laut Ekici et al. dafür.

Dr. med. Susanne Krome, Melle 